\title{
The Effects of Clozapine on Symptom
} Reduction, Neurocognitive Function, and Clinical Management in Treatment-Refractory State Hospital Schizophrenic Inpatients

\author{
Anne L. Hoff, Ph.D., William O. Faustman, Ph.D., Mary Wieneke, Ph.D., Scott Espinoza, \\ Martin Costa, Ph.D., Owen Wolkowitz, M.D., and John G. Csernansky, M.D.
}

Thirty chronically hospitalized, refractory schizophrenic patients were evaluated while on typical neuroleptics and again after 12 weeks of clozapine treatment. Patients demonstrated small but statistically significant reductions in total Brief Psychiatric Rating Scale (BPRS) symptoms, need for seclusion and restraint, and PRN medications, and they frequently were transferred to a less restrictive treatment environment. Neuropsychological test data from a subset of patients suggested improvement on measures of verbal fluency and graphomotor speed, but deterioration on measures of visual memory and executive/frontal ability. Clozapine's different effects on multiple neurotransmitter systems may be responsible for its mixed effects on cognitive abilities. No significant relationships were found between symptom reduction, cognitive improvement, and transfer to a less restrictive environment.

[Neuropsychopharmacology 15:361-369, 1996]
KEY WORDS: Cognition; Clozapine; Schizophrenia

Clozapine is an atypical antipsychotic neuroleptic that has been shown to be more effective than conventional neuroleptics for the treatment of refractory schizophrenic patients (Kane et al. 1988). Clozapine is characterized by its relatively stronger blockade of $5-\mathrm{HT}_{2}$ serotonin and $D_{1}$ dopamine receptors and weaker blockade of $\mathrm{D}_{2}$ receptors. In addition, it is an antagonist at adren-

From the Biological Psychiatry Treatment and Research Center (ALH, MW, SE, MC), Napa State Hospital, Napa, CA; the Veterans Affairs Palo Alto Health Care System (WOF), Palo Alto Division, Palo Alto, CA; the Department of Psychiatry and Behavioral Sciences (ALH, WOF), Stanford University School of Medicine, Stanford, CA; the Department of Psychiatry (OW), University of CaliforniaSan Francisco, San Francisco, CA; and the Department of Psychiatry (JGC), Washington University School of Medicine, St. Louis, MO.

Address correspondence to: Anne L. Hoff, Ph.D., Biological Psychiatry Treatment and Research Center, Napa State Hospital, 2100 Napa-Vallejo Highway, Napa, CA 94558.

Received May 9, 1995; revised October 24, 1995; accepted November 15, 1995. ergic and muscarinic receptors (Meltzer 1994). It produces fewer extrapyramidal side effects than typical neuroleptics (Kane et al. 1988; Pickar et al. 1992), improves measures of quality of life (Meltzer et al. 1990), reduces hospital readmissions (Breier et al. 1993), and reduces the need for seclusion and restraint (Chiles et al. 1994). Weekly blood monitoring is necessary to reduce the risk of agranulocytosis, which occurs in approximately 1 to $2 \%$ of patients (Kane et al. 1988; Lieberman et al. 1988).

Relative to the plethora of studies on the biochemistry and clinical efficacy of clozapine, there have been few studies on the effects of clozapine on neurocognitive function. Goldberg et al. (1993) compared the cognitive performance of 15 treatment-refractory patients (13 diagnosed with chronic schizophrenia, 1 schizoaffective, and 1 psychosis not otherwise specified [NOS]) on conventional neuroleptics, with or without adjunctive lithium or carbamazapine, to their performance on clozapine, with or without similar adjunctives. A com- 
prehensive neuropsychological test battery, including intellectual, memory, attentional, executive, and spatial measures, was administered at baseline and 3 to 24 months after clozapine treatment (average of 15 months). Despite significant reductions in clinical symptomatology, there was no significant improvement on any of the neuropsychological measures and significantly worse performance on a measure of short-term visual memory. The strength of this study was in the measurement of the same patient at two time points, during treatment with conventional neuroleptics and then after clozapine. Its relative weaknesses were the variable length of time for retesting and the fact that a variety of adjunctive drugs was also used at both time points. Hagger et al. (1993) also compared the cognitive performance of 36 treatment-refractory schizophrenic patients at baseline ( 27 were drug free, 5 on conventional neuroleptics, and 4 on 25 to $100 \mathrm{mg}$ of clozapine for 1 to 3 days) and again after 6 weeks and 6 months of clozapine monotherapy. In this study, patients performed significantly better on a verbal fluency task after 6 weeks and on the same verbal fluency task and the Digit Symbol test, a measure of attention and graphomotor speed, after 6 months of clozapine treatment.

The effects of both typical and atypical neuroleptics on cognitive function remain unclear; however, chronic administration of typical neuroleptics appears consistently to improve attentional function and some aspects of visuomotor performance in schizophrenic patients (Cassens et al. 1990; King 1990; Faustman and Hoff 1996). Thus, comparing the performance of patients while on clozapine to their drug-free state may not reveal much about the unique properties of clozapine compared with other drugs in ameliorating the cognitive deficits of schizophrenia.

To determine the relative effects of clozapine versus typical neuroleptics on cognitive measures, an unblinded, parallel group study of non-treatment-resistant schizophrenic patients was conducted by Lee et al. (1994) using the same neuropsychological tests as Hagger et al. (1993). Patients were randomly assigned to typical neuroleptics plus benztropine where needed $(n=23)$ versus clozapine treatment $(n=24)$ and then tested at a drug-free baseline after 6 weeks, 6 months, and 12 months of drug treatment. At 6 months, performance on 7 of 9 neuropsychological tests was significantly improved compared to baseline, whereas performance on only 1 of 9 tests was significantly improved in the typical neuroleptic group. At 12 months, the number of tests showing significant improvement in each group was more comparable, with 4 in the clozapine group and 2 in the typical treatment group. When the two treatment groups were compared directly, the clozapine group showed superior performance to the typical group on two measures of cognitive function, the Controlled Oral Word Association, a measure of verbal fluency, and the Digit Symbol test. Significant differences between in the two groups existed at 6 weeks and at 6 and 12 months on these two tests.

In a double-blind, parallel-group comparison of partially responsive neuroleptic patients, Buchanan et al. (1994) assessed 19 schizophrenic patients on clozapine (400 mg daily) and 19 patients on haloperidol $(20 \mathrm{mg}$ daily) after 10 weeks. In both cases, cognitive performance on the study medication was compared to test performance during a 6-week baseline period of 10 to $30 \mathrm{mg}$ fluphenzapine. Thirty-three of these patients were also evaluated after subsequent treatment with clozapine after 1 year. After 10 weeks of treatment, haloperidol-treated patients did significantly worse on the Category Fluency and Block Design tests than did the clozapine-treated patients. The performance of the clozapine patients remained the same before and during treatment. There were no statistically significant differences between the two groups on 11 other tests. After 1 year of open-label clozapine treatment, there were significant improvements in verbal fluency (Controlled Oral Word Association) and two measures of visuospatial ability (Block Design and Mooney Faces Closures) compared with baseline performance with fluphenazine treatment. However, performance on Trails B, a measure of cognitive flexibility, concentration, and motor speed, was significantly worse.

In summary, it appears that when clozapine treatment has been compared to treatment with typical neuroleptics, patients demonstrate slight improvements on tasks of verbal fluency and graphomotor speed. However, there is also evidence that clozapine-treated patients perform worse on tests of visual memory and cognitive flexibility, that is, the ability to switch cognitive sets.

The purpose of the present study was to compare the efficacy of clozapine and conventional antipsychotics on cognitive function and to relate these differences, if present, to other effects on symptom reduction and clinical management factors in a refractory population of schizophrenics hospitalized in a chronic setting. As cognitive disability has been suggested as a rate-limiting factor in the rehabilitation of schizophrenics (Goldberg et al. 1993), we hoped that improvements in cognitive function in a refractory population would relate to improvement in clinical state and in the ability of staff to place patients outside of the institution. We evaluated 30 schizophrenic patients who were on typical neuroleptics and then again after 12 weeks of clozapine treatment. In 20 of these patients, neuropsychological testing was performed at baseline and after clozapine treatment. Our hypotheses were that patients on clozapine would demonstrate (1) significant reductions in clinical symptomatology; (2) reductions in the need for PRN medications and behavioral restraints; and (3) improvements on measures of verbal fluency 
(Controlled Oral Word Association), graphomotor speed (Symbol Digit Modalities Test), and motor speed (Finger Tapping); and (4) deterioration on measures of visual memory (Visual Reproduction, Benton Visual Retention Test) and on measures that require the shifting of mental sets (Wisconsin Card Sort, Trails B). We also hypothesized that cognitive improvement would be common in patients transferred to less restrictive treatment settings.

\section{METHODS}

\section{Subjects}

The study took place at Napa State Hospital (NSH), a state hospital in Northern California with approximately 800 patients. Thirty male patients were recruited by consulting a list of patients provided by a hospital therapeutic review committee, which approved requests from hospital physicians to start their patients on clozapine. Patients were eligible for a clozapine trial at Napa State Hospital beginning in December 1991 after they met specific statewide criteria: (1) tardive dyskinesia; (2) intractable extrapyramidal side effects; or (3) documented evidence of poor response to at least two conventional neuroleptics from different chemical classes given for a minimum of 6 weeks in daily doses equivalent to at least $1,000 \mathrm{mg}$ of chlorpromazine. Sixty-one patients were asked to participate in the study. Twentyeight patients refused and three patients had legal conservators who refused to allow their conservatee to participate, leaving a total of 30 patients who participated.

Of the 30 patients studied, all evidenced poor response to two previous neuroleptics. In addition, 17 of 30 patients had tardive dyskinesia or intractable extrapyramidal side effects. Patients for this study were evaluated between June 1992 and January 1994.

Of these 30 patients, 20 patients received neuropsychological testing while on typical neuroleptics and again after 12 weeks of clozapine treatment. The other 10 patients could not be tested because of lack of cooperation ( $n=9$ ) or language difficulties (one patient's native language was Greek).

Ten of the 30 patients were diagnosed by two independent psychiatrists, one using the Structured Clinical Interview for DSM-II-R (Spitzer et al. 1992) and the other a DSM-III-R checklist. Final diagnosis was reached by consensus. In the remaining patients, DSMIII-R diagnoses were made after reviewing the most recent hospital charts. Ten of 20 patients who received neuropsychological testing were diagnosed using the consensus of the SCID and DSM-III-R checklist. Age of onset was defined as the age at which any acute symptom required for the diagnosis of schizophrenia in the DSM-III-R manual first appeared. Duration of illness was then calculated as the current age minus the age of onset. All patients were male.

\section{Procedure}

Patients identified for the study gave informed consent after receiving an explanation of the purpose and procedures involved in the study. Permission for participation was also obtained from patients' conservators, where appropriate. Patients were then given a battery of neuropsychological tests designed to tap a wide variety of cognitive functions previously shown by our group to be impaired in schizophrenic patients (Hoff et al. 1992a; 1992b). All patients were tested and evaluated while in the hospital and were located on a variety of units throughout the hospital. Other than receiving neuropsychological testing and Brief Psychiatric Rating Scale (BPRS) ratings, their treatment was the same as patients not in this study.

Patients were first tested while receiving drug treatment considered optimal by their treating psychiatrist and prior to any changes required in anticipation of switching to clozapine. Optimal treatment was defined as the treatment that their attending psychiatrist had chosen prior to the clozapine trial and was considered optimal in light of the other medication trials that had already been tried. Nineteen of the 20 patients were taking typical neuroleptics. Of these 19, 6 patients were also taking lithium, 2 patients were taking carbamazepine, and 1 patient was taking lithium and carbamazepine. In addition, 3 patients were taking clonazepam, 2 patients were taking alprazolam, 2 were taking diazepam, 1 was taking propanolol, and 1 patient was taking lorazepam. Eight patients were taking benztropine mesylate, and 1 patient was on trihexyphenidyl. On average, the patients (excluding the patient on no neuroleptic drug) received a daily chlorpromazine equivalency of $1,418.4 \pm 809.9 \mathrm{mg}$ while on typical neuroleptics. The one patient who was not taking neuroleptics was treated with a combination of clonazepam, diazepam, lorazepam, and propanolol. He is included in this group because this was his optimal treatment prior to the administration of clozapine.

Two estimates of general intellectual functioning were administered, the Pro-rated Verbal IQ from the Wechsler Adult Intelligence Scale (adapted from Satz and Mogel 1962) and a standard score equivalent from the Reading subtest of the Wide Range Achievement Test (Jastak and Wilkinson 1984). Measures of shortterm verbal recall included the Logical Memory (paragraphs) and Associate Learning (word pairs) subtests from the Wechsler Memory Scale (Wechsler 1945) and the California Verbal Learning Test (CVLT; number of words recalled on trial 5; Delis et al. 1987). Short-term visual memory was measured by the Visual Reproduction subtest of the Wechsler Memory Scale and the 
number of errors from the Benton Visual Retention Test (BVRT) (Benton 1974). Executive/frontal functioning was assessed using the Wisconsin Card Sorting Test (Heaton 1981). Measures of expressive speech included the Boston Naming Test (Goodglass and Kaplan 1983), a measure of confrontation naming and the Controlled Oral Word Association (Benton and Hamsher 1989), a measure of verbal fluency. Symbol Digit Modalities Test-Written (Smith 1973) and Trails B (Reitan 1979) were given to assess mental processing speed, concentration, and graphomotor speed. Pure motor speed was measured by the Finger Tapping Test (Reitan 1979). Before and after 12 weeks of clozapine treatment, patients were tested using alternate forms of the tests where they existed (memory tests and the verbal fluency test).

Patients' symptoms were assessed at both time points using the 18-item ( 1 indicates absent and 7 indicates severe) BPRS (Overall and Gorham 1962). The mean scores of two trained research assistants who attended a single interview were averaged. BPRS interrater reliability in our center is 0.80 to 0.90 for the total score. BPRS ratings were available for 28 of 30 patients, including 18 of the patients who received neuropsychological testing.

A BPRS Positive Symptom score was calculated as the average of the hallucinatory behavior, unusual thought content, and conceptual disorganization items. A BPRS Negative System score was calculated as the average score of the blunted affect, emotional withdrawal, and motor retardation items (Thiemann et al. 1987; Faustman 1994). The BPRS activation score was the average of the tension, mannerisms and posturing, and excitement items and the Hostile-Suspiciousness scale was the average of the hostility, suspiciousness, uncooperativeness, and grandiosity items (Hedlund and Vieweg 1980).

Clinical management variables included (1) the number of seclusion and restraint orders (S\&Rs); and (2) the number of non-neuroleptic and neuroleptic PRN drugs given for agitation 3 months before clozapine administration and 3 months following the start of clozapine. The patients' living arrangements (intensity of supervision) before and after clozapine were rated using the following scale: 0 , locked unit at NSH; 1 , open (unlocked) unit at NSH; 2, locked residential facility; 3, open residential facility (i.e., board and care home, group home); 4 , living with family members; and 5, living independently.

\section{Statistical Analyses}

Predicted changes in neuropsychological test scores, BPRS variables, and clinical management variables were tested by paired $t$ tests, using a one-tailed significance level. Effect sizes for neuropsychological test variables in which there were no a priori hypotheses were calculated by dividing the paired $t$ score for those mean differences by the square root of the sample size. In essence, the effect size is the average difference score divided by the standard deviation of the difference scores and thus is independent of sample size (Kraemer and Thiemann 1987). It provides an interpretable estimate of the magnitude of a difference, without the drawbacks of performing significance tests when there are no a priori hypotheses. Difference scores of all variables were calculated by substracting 12-week scores from baseline scores. The relationships between difference scores were estimated using Spearman rho correlations.

\section{RESULTS}

The demographic and clinical characteristics of the patient cohort are described in Table 1. The population was a chronically ill, continuously hospitalized, group of patients with a relatively early age of onset. Total BPRS scores for this group were $62.5 \pm 8.9$. They had been ill for approximately 18 years and had been continuously hospitalized at NSH for the past $7.5 \pm 5.3$ years. After 12 weeks of treatment, they were receiving $667.5 \pm 163.5$ (range $=425$ to 900 ) $\mathrm{ng}$ of clozapine monotherapy. Overall intellectual functioning at baseline was estimated to be in the low average range by using WRAT-Reading scores, where the standard score equivalent at baseline was $87.2 \pm 13.1$. Reading pronounciation measures were used to estimate premorbid intellectual functioning as this ability tends to be least affected by known organic brain insults (Nelson and McKenna 1975).

As predicted, there were statistically significant reductions on the Total BPRS $(t=-2.35, p<.01)$, Activation $(t=1.95, p<.03)$, and Hostility/Suspiciousness $(t=-1.68, p<.05)$ scales (see Table 2). Patients also received significantly fewer episodes of restraint and seclusion after clozapine treatment $(t=-2.03, p<.03)$ and fewer non-neuroleptic PRN drugs (primarily lorazepam;

Table 1. Demographic and Clinical Characteristics of Patient Sample $(n=30)$

\begin{tabular}{lr}
\hline & Mean \pm SD \\
\hline Age (years) & $35.4(7.4)$ \\
Education (years) & $9.9(3.2)$ \\
Age of onset (years) & $18.0(4.4)$ \\
Age of first hospitalization & $19.9(4.5)$ \\
Duration of illness (years) & $17.9(8.0)$ \\
Length of most recent & \\
$\quad$ hospitalization (years) & $7.5(5.3)$ \\
Diagnosis subtype & \\
$\quad$ Undifferentiated & 13 \\
$\quad$ Paranoid & 8 \\
Disorganized & 9 \\
\hline
\end{tabular}


Table 2. Symptom and Outcome Measures

\begin{tabular}{|c|c|c|c|c|}
\hline & $\begin{array}{c}\text { Typical } \\
\text { Neuroleptics } \\
(n=30 \\
\text { mean } \pm \text { SD })^{a}\end{array}$ & $\begin{array}{c}\text { Clozapine } \\
(n=30 \\
\text { mean } \pm \text { SD })\end{array}$ & $t$ & $\underset{\text { (one tail) }}{p}$ \\
\hline \multicolumn{5}{|l|}{$\operatorname{BPRS}(n=28)$} \\
\hline Total & $62.5 \pm 8.9$ & $58.3 \pm 7.1$ & -2.35 & 0.01 \\
\hline Positive & $4.9 \pm 0.9$ & $4.8 \pm 0.8$ & -0.39 & 0.35 \\
\hline Negative & $3.1 \pm 1.1$ & $2.9 \pm 0.2$ & -0.99 & 0.16 \\
\hline Activation & $2.8 \pm 0.6$ & $2.5 \pm 0.6$ & -1.95 & 0.03 \\
\hline Hostility/suspiciousness & $3.7 \pm 0.8$ & $3.3 \pm 0.6$ & -1.68 & 0.05 \\
\hline Number of S\&R orders & $2.4 \pm 4.8$ & $0.8 \pm 1.5$ & -2.03 & 0.03 \\
\hline \multicolumn{5}{|l|}{ Number of PRN's } \\
\hline (non-neuroleptic) & $11.8 \pm 17.6$ & $7.4 \pm 10.8$ & -1.91 & 0.03 \\
\hline \multicolumn{5}{|l|}{ Number of PRN's } \\
\hline (neuroleptic) & $2.0 \pm 6.4$ & $2.2 \pm 7.3$ & 0.12 & 0.45 \\
\hline Number of PRN's (both) & $13.8 \pm 17.4$ & $9.5 \pm 11.9$ & -1.67 & 0.05 \\
\hline
\end{tabular}

${ }^{a}$ One patient had non-neuroleptic combination treatment (see methods).

$t=-1.91, p<.03)$ and total PRN drugs $(t=-1.67, p<$ .05) for agitation. However, using the Kane et al. (1988) criteria for treatment responsiveness $(20 \%$ reduction intotal BPRS score), only 4 of $28(14 \%)$ would have been classified as clozapine responders.

After clozapine treatment, 10 patients remained on a locked NSH unit, 8 went to an open (unlocked) NSH unit, 10 went to a locked residential facility in the community, and 2 were discharged to an open residential facility (board and care home). In summary, 18 patients remained at NSH and 12 were discharged. Of the $30 \mathrm{pa}-$ tients, 25 remain on clozapine as of the time of writing this paper. Five patients were taken off clozapine ( 1 patient developed granulocytopenia, 1 developed an arrhythmia, 1 had sleep difficulties and hypotension, the other 2 were treatment failures).

Neuropsychological test performance (see Table 3) indicated that patients performed significantly better on the Controlled Oral Word Association Test $(t=2.34, p<$ $.02)$, which measures the ability to generate words beginning with the letters C, F, and L. Patients also improved significantly on the Symbol Digit Modalities Test (written, $t=3.01, p<.004$ ) and on the Finger Tapping Test $(t=2.5, p<.01)$, which both involve a speeded motor response. In contrast, patients tested with clozapine made more errors on the Benton Visual Retention Test, which requires drawing geometric designs after a 10-second exposure $(t=2.08, p<.03)$. They also performed significantly worse on the Wisconsin Card Sorting Test in achieving the number of correct categories $(t=2.02, p<.03)$, suggesting that they had greater difficulty in maintaining the correct concept on a problem-solving task, that is, greater frontal lobe dysfunction (Heaton 1981). Small to medium effect sizes were found for performances on the other neuropsychological tests ( -.18 to .58$)$. The largest effect size was found on the California Verbal Learning Test, which measures an individual's ability to recall a 16-item list after the list is repeated five times. The effect size was in the direction of improvement. In summary, relative to typical neuroleptics, clozapine had various effects on cognitive abilities, causing improvement in some and deterioration in others.

Difference scores for positive, negative, and total BPRS symptoms, S\&Rs, and PRNs (baseline minus 12 weeks) were correlated with difference scores for only those neuropsychological tests $(n=5)$ that significantly changed after clozapine $(p<.05)$. Reduction in total BPRS symptoms was associated with improvement on the Controlled Oral Word Association (rho $=-.53, p<$ .04), Symbol Digit Modalities Test (rho $=-.64, p<$ .005 ), and Finger Tapping (rho $=-.54, p<.03$ ). The other correlations were not statistically significant. Repeated-measures analyses of variance (ANOVAs) were then used to compare neuropsychological test results in the group of discharged patients $(n=9)$ with those continuing as NSH inpatients $(n=11)$. There were no statistically significant group-by-time interactions. It should be noted, however, that group sizes were very small.

To determine whether reductions in psychiatric symptoms were related to patient disposition, BPRS, S\&R, and PRN change scores were correlated with the living arrangement score. Again, no statistically significant relationships emerged. Patients who were discharged $(n=12)$ were also compared to those who were not $(n=18)$ using baseline and 12-week BPRS summary scale scores and repeated-measures ANOVA. Again, there were no significant group or group-by-time interactions, suggesting no pattern of symptom improvement related to patient outcome.

Within the group of patients who stayed at NSH $(n=$ 18), patients who were transferred to an unlocked unit $(n=8)$ had initial lower preclozapine levels of seclusion and restraint $(t=-2.48, p<.025)$ and need for PRN 
Table 3. Neuropsychological Test Scores on Typical Neuroleptics And After 12 Weeks of Clozapine

\begin{tabular}{|c|c|c|c|c|}
\hline $\begin{array}{l}\text { Cognitive Measures } \\
\text { (predicted direction) }\end{array}$ & $\begin{array}{l}\text { Typical Neuroleptics } \\
(n=20, \text { mean } \pm S D)^{a}\end{array}$ & $\begin{array}{c}\text { Clozapine } \\
(n=20, \text { mean } \pm \text { SD })\end{array}$ & $t$ & $\underset{\text { (one tail) }}{p}$ \\
\hline Benton Visual Retention Test, & $14.6 \pm 4.6$ & $17.4 \pm 5.7$ & 2.08 & 0.03 \\
\hline Visual Reproduction & $4.7 \pm 3.4$ & $4.9 \pm 3.2$ & 0.26 & 0.40 \\
\hline \multicolumn{5}{|l|}{ Wisconsin Card Sorting Test } \\
\hline Number of categories & $2.1 \pm 2.3$ & $1.3 \pm 1.7$ & -2.02 & 0.03 \\
\hline Number of total errors & $69.5 \pm 21.8$ & $73.3 \pm 23.7$ & 1.41 & 0.09 \\
\hline Number of pers. resp. & $58.1 \pm 36.6$ & $54.7 \pm 33.9$ & -0.28 & 0.39 \\
\hline Controlled Oral Word Association & $25.0 \pm 11.7$ & $31.7 \pm 13.0$ & 2.34 & 0.02 \\
\hline Symbol Digit Modalities-written & $19.2 \pm 9.8$ & $24.5 \pm 10.2$ & 3.01 & 0.004 \\
\hline Trails B, number of Seconds & $241.3 \pm 106.6$ & $251.9 \pm 102.3$ & 0.23 & 0.41 \\
\hline Finger Tapping $(\mathrm{R}+\mathrm{L}) / 2$ & $38.1 \pm 7.4$ & $42.4 \pm 4.4$ & 2.50 & 0.01 \\
\hline $\begin{array}{l}\text { Cognitive Measures } \\
\text { (no prediction) }\end{array}$ & & & \multicolumn{2}{|c|}{ Effect Size } \\
\hline WAIS-R Verbal IQ (Pro rated) & $77.8 \pm 13.2$ & $79.8 \pm 14.1$ & \multicolumn{2}{|c|}{0.22} \\
\hline WRAT-Reading & $87.2 \pm 13.1$ & $82.7 \pm 17.0$ & \multicolumn{2}{|c|}{-0.18} \\
\hline Logical Memory $(A+B)$ & $5.3 \pm 4.2$ & $7.0 \pm 4.5$ & \multicolumn{2}{|c|}{0.37} \\
\hline Associative Learning & $8.2 \pm 5.3$ & $6.1 \pm 3.0$ & \multicolumn{2}{|c|}{-0.39} \\
\hline California Verbal Learning Test & $5.0 \pm 3.1$ & $6.1 \pm 3.0$ & \multicolumn{2}{|c|}{0.58} \\
\hline Boston Naming Test & $42.1 \pm 13.0$ & $45.6 \pm 10.8$ & \multicolumn{2}{|c|}{0.43} \\
\hline
\end{tabular}

${ }^{a}$ One patient had non-neuroleptic combination treatment (see methods).

medications $(t=-2.21, p<.04)$ than those who were maintained on a locked unit $(n=10)$.

\section{DISCUSSION}

Compared to patients on typical neuroleptics, continuously hospitalized patients who received 12 weeks of clozapine treatment had significantly fewer psychiatric symptoms, required fewer periods of seclusion and restraint, required fewer PRN medications for agitation, and were frequently transferred to a less restrictive treatment setting. As predicted, patients improved on cognitive measures of graphomotor speed and verbal fluency. Although reductions in symptoms were related to some areas of cognitive improvement, there was no relationship between the disposition of patients and cognitive improvement.

Comparing our patients to those of the Kane et al. (1988) study, it should be noted that our patients had a $14 \%$ response rate compared to their $30 \%$ response rate for refractory symptoms. Our patients had comparable BPRS total scores to those in the Kane et al. (1988) and had similar ages of first hospitalization and thus, similar durations of illness. However, the length of the most recent hospitalization for our patients was $7.5 \pm 5.3$ years, while for the Kane et al. (1988) group, it was approximately 4.2 years. In addition, a large proportion of patients from the Kane et al. study were from Veterans Administration medical centers, whereas our patients were state hospital patients. Virtually all patients are hospitalized at Napa State Hospital after a history of failure at all other mental health facilities and because of serious behavorial management problems, primarily assaultiveness. Thus, our patients may represent a severity of illness and/or treatment resistance even greater than that of patients in the Kane et al. study. In spite of this relatively poor response rate as defined by Kane et al. criteria, other outcome measures were positively affected by clozapine, supporting the notion of multidimensional assessment in evaluating the effects of clozapine (Meltzer 1992).

Two major changes in the hospital administration may have influenced patient disposition independent of change in clinical state, the opening of transitional unlocked units at the hospital and the transfer of patients out of the institution by nearby counties for fiscal reasons. As a result, we determined patient disposition for a comparison group of the 53 chronic schizophrenic patients who were located on the same units during the same period of time as our target group of 30 clozapine treated patients. These were patients who did not receive clozapine. This information was obtained from a review of unit log books that track the census of the unit and the setting to which patients are discharged. Of the 30 patients who were treated with clozapine, $20(67 \%)$ were transferred to a less restrictive setting, either assigned to an unlocked NSH unit or discharged. Of the 53 patients who did not receive clozapine, $23(43 \%)$ were transferred to a less restrictive setting (Chi- 
square $=4.15, p<.042)$. Thus, it appears that the transfer of the clozapine patients to less restrictive settings was not accounted for solely by hospital administrative changes and that clozapine patients were more likely to require less intensity of supervision.

Our findings are consistent with those of previous studies (Lee et al. 1994) in which clozapine treatment was related to improved performance on tests of graphomotor speed and verbal fluency compared with conventional neuroleptics. In our study, patients performed better on the Symbol Digit Modalities Test (SDMT) and the Controlled Oral Word Association test. The SDMT requires the individual to copy numbers in association to symbols based on a key of paired numbers and symbols given at the top of the page. It is a variation of the Digit Symbol test, on which patients also performed better during clozapine treatment as reported in other studies (Buchanan et al. 1994; Lee et al. 1994). Both the Digit Symbol test and the SDMT require the ability to concentrate and mental processing speed and motor speed (i.e., writing numbers during a 90 -second period). Goldberg and Weinberger (1994) have suggested that these tasks involve a "chronometry" of action, functions that have been linked to the basal ganglia. Because clozapine has weaker dopamine receptor blockade in these areas (Farde et al. 1992), they speculate that these "effects" represent the absence of typical neuroleptic side effects (i.e., slowing of mentation and motor speed). They also assert that clozapine's blockade of $D_{1}$ receptors in the frontal cortex may relate to clozapine's negative effects on the Wisconsin Card Sort. However, clozapine's effects on a variety of other neuroreceptors (e.g., muscarinic) might also explain such findings. Whatever the explanation, our data support the hypothesis that clozapine adversely effects executive/ frontal functioning at least on a short-term basis.

Like Goldberg et al. (1993), we found that patients on clozapine also demonstrated worse performance on a measure of short-term visual memory for geometric designs. If this decline related to clozapine's strong antimuscarinic properties, one would expect a similar decline in verbal memory performance. However, the effect sizes for verbal memory tasks in this study suggest improvement on two tasks and decline on another, the Associative Learning subtest of the Wechsler Memory Scale, which involves word-pair recall. Therefore, the results are mixed with regard to clozapine's effects on verbal memory.

In summary, clozapine had a variety of beneficial effects in severely ill, continuously hospitalized patients. These effects included improvements in symptoms and some cognitive functions, particularly those requiring rapid mental processing and motor output. Furthermore, clozapine facilitated the transfer of patients to less restrictive settings, and $30 \%$ of the patients were discharged despite the fact that on average these pa- tients had been continuously hospitalized at Napa State Hospital for the last $7^{1 / 2}$ years. There were no demonstrable relationships between symptom reduction or cognitive improvement and patient disposition. However, such a relationship may have been obscured by other factors changing in the hospital and a restriction in range (floor effect) in terms of patient disposition and clinical symptoms.

The limitations of this study are that treatment was open-label, the follow-up period limited, and the sample was restricted in size, thus reducing power in detecting differences. Notably, some recent data indicated that clozapine may continue to improve patients' symptoms for 9 to 12 months or more (Meltzer 1992). In addition, several other studies (Hagger et al. 1993; Buchanan et al. 1994; Lee et al. 1994) have demonstrated improvement in cognitive abilities after 6 months and 12 months of clozapine treatment, although at least in one study, there were fewer differences from basline at 12 months than at 6 months (Lee et al. 1994). It is conceivable that our study would have found greater improvement in cognitive abilities as a result of clozapine if the evaluation period had been longer.

Another weakness is that our comparison of clozapine treatment to typical neuroleptic treatment may be somewhat blurred by the fact that a number of our patients were on combination treatments at baseline. It is conceivable that patients improved cognitively on some measures simply because they were on fewer medications at second testing. However, examination of the differences on neuropsychological testing between baseline and clozapine treatment for patients on baseline neuroleptic alone (plus benztropine, $n=10$ ) versus patients on combination treatments (neuroleptic, benztropine, and additional medication such as lithium, $n=9$ ) revealed that both groups changed on tests in the same direction and to the same degree. Naturally, these sample sizes are too small for meaningful statistical testing, but it does not appear that the group of patients starting with combination treatments alone was accounting for the differences seen.

The strengths of the study were that we compared the cognitive performance of a group of patients on typical neuroleptics chosen as "optimal treatment" by their physicians with their performances on clozapine, after a consistent interval. This within-subjects design has the advantage of controlling extraneous sources of individual subject variance common to between-subjects designs. Moreover, our study design closely reflected the circumstances of real clinical care given to such patients, in that these patients are rarely taken off all medications.

A more optimal research design would have been to begin treatment for all patients using a low potency typical neuroleptic for 6 months and then blindly switch half of them to clozapine for a second 6 month 
period. However, that sort of study would have been impossible to do in our setting because of the need for weekly blood monitoring in the "control" group and because the blind would be difficult to realize given the sedating nature of clozapine in the first several of weeks of treatment. Nevertheless, teasing out the acute and more chronic effects of typical and an atypical neuroleptic such as clozapine on cognition could be accomplished in this fashion. In the future, we hope to undertake such studies. In the meantime, our results do confirm a somewhat unique profile of cognitive improvement and deterioration associated clozapine. Future studies will examine activation, with functional MRI, of brain regions during these cognitive tasks while patients are on clozapine and on typical neuroleptics. These kinds of studies may further our understanding of clozapine's unique mechanism of action.

\section{ACKNOWLEDGMENTS}

The authors wish to thank Sue Thiemann, M.S., for her statistical consultation and Thomas Nordahl, M.D., and William Kremen, Ph.D., for their assistance on the manuscript. Research supported by the Department of Mental Health, State of California, Napa State Hospital, the Department of Veterans Affairs, and grant MH30854 to Adolf Pfefferbaum, M.D., National Institute of Mental Health.

\section{REFERENCES}

Benton AL (1974): The Revised Visual Retention Test, ed. 4. New York, Psychological Corporation

Benton AL, Hamsher K de S (1989): Multilingual Aphasia Examination. Iowa City, University of Iowa, AJA Associates

Breier A, Buchanan RW, Irish D, Carpenter WT Jr (1993): Clozapine treatment of outpatients with schizophrenia: Outcome and long-term response patterns. Hosp Comm Psychiatry 44:1145-1149

Buchanan RW, Holstein C, Brier A (1994): The comparative efficacy and long-term effect of clozapine treatment on neuropsychological test performance. Biol Psychiatry 36:717-725

Cassens G, Inglis AK, Appelbaum PS, Gutheil TG (1990): Neuroleptics: Effects on neuropsychological function in chronic schizophrenic patients. Schiz Bull 16:477-499

Chiles JA, Davidson P, McBride D (1994): Effects of clozapine on use of seclusion and restraint at a state hospital. Hosp Comm Psychiatry 45:269-271

Delis D, Kramer J, Kaplan E, Ober B (1987): California Verbal Learning Test: Research Edition. San Antonio, Psychological Corporation

Farde L, Nordstrom A, Wiesel F, Pauli S, Halldin C, Sedvall G (1992): Positron emission tomographic analysis of central $D_{1}$ and $D_{2}$ dopamine receptor occupancy in patients treated with classical neuroleptics and clozapine. Arch Gen Psychiatry 49:538-544
Faustman WO (1994): Brief Psychiatric Rating Scale. In Maruish ME (ed), The Use of Psychological Testing for Treatment Planning and Outcome Assessment. Hillsdale, NJ, Erlbaum, pp 371-401

Faustman WO, Hoff AL (1996): Effects of antipsychotic drugs on neuropsychological measures. In Csernansky JG (ed), Handbook of Experimental Pharmacology: Antipsychotics. Berlin, Springer-Verlag, pp 445-477

Goldberg TE, Weinberger DR (1994): The effects of clozapine on neurocognition. J Clin Psychiatry (suppl B) 55:88-90

Goldberg TE, Greenberg RD, Griffin SJ, Gold JM, Kleinman JE, Pickar D, Schultz SC, Weinberger DR (1993): The effect of clozapine on cognition and psychiatric symptoms in patients with schizophrenia. Brit J Psychiatry 162:43-48

Goodglass H, Kaplan E (1983): The Assessment of Aphasia and Related Disorders. Philadelphia, Lea \& Febiger

Hagger C, Buckley P, Kenny JT, Friedman L, Ubogy D, Meltzer HY (1993): Improvement in cognitive functions and psychiatric symptoms in treatment-refractory schizophrenic patients receiving clozapine. Biol Psychiatry 34:702-712

Heaton RK (1981): Wisconsin Card Sorting Test (WCST). Manual. Odessa, Florida, Psychological Assessment Resources.

Hedlund JL, Vieweg BW (1980): The Brief Psychiatric Rating Scale (BPRS): A comprehensive review. J Oper Psychiatry 11:48-64

Hoff AL, Riordan H, O'Donnell D, Morris L, DeLisi LE (1992a): Neuropsychological functioning of first-episode schizophreniform patients. Am J Psychiatry 149:898-903

Hoff AL, Riordan H, O'Donnell D, Stritzke P, Neale C, Boccio A, Anand A, DeLisi LE (1992b): Anomalous lateral sulcus asymmetry and cognitive function in first-episode schizophrenia. Schiz Bull 18:257-272

Jastak S, Wilkinson G (1984): The Wide Range Achievement Test-Revised. Wilmington, DE, Jastak Associates

Kane J, Honigfeld G, Singer J, Meltzer H, Clozaril Collaborative Study Group (1988): Clozapine for the treatmentresistant schizophrenic: A double-blind comparison with chloromazine. Arch Gen Psychiatry 45:789-796

King DJ (1990): The effect of neuroleptics on cognitive and psychomotor function. Brit J Psychiatry 157:799-811

Kraemer H, Thiemann S (1987): How Many Subjects? Statistical Power Analysis in Research. Newbury Park, CA, Sage

Lee MA, Thompson PA, Meltzer HY (1994): Effects of clozapine on cognitive function in schizophrenia. J Clin Psychiatry (suppl B) 55:82-87

Lieberman JA, Johns CA, Kane JM et al. (1988): Clozapineinduced agranulocytosis: Noncross reactivity with other psychotropic drugs. J Clin Psychiatry 49:271-277

Meltzer HY (1992): Dimensions of outcome with clozapine. Br J Psychiatry (suppl. 17) 160:46-53

Meltzer HY (1994): An overview of the mechanism of action of clozapine. J Clin Psychiatry (suppl B) 55:47-52

Meltzer HY, Burnett S, Bastani B, Ramirez LF (1990): Effects of six months of clozapine treatment on the quality of life of chronic schizophrenic patients. Hosp Comm Psychiatry 41:892-897 
Nelson HE, McKenna P (1975): The use of reading ability in the assessment of dementia. Brit J Soc Clin Psychol 14:259-267

Overall JE, Gorham DR (1962): The Brief Psychiatric Rating Scale. Psych Reports 10:799-812

Pickar D, Owen RR, Litman RE, Konicki E, Gutierrez R, Rapaport MH (1992): Clinical and biologic response to clozapine in patients with schizophrenia: Crossover comparison with fluphenazine. Arch Gen Psychiatry 49:345-353

Reitan RM (1979): Manual for Administration of Neuropsychological Test Batteries for Adults and Children. Tucson, AZ, Ralph M. Reitan
Satz P, Mogel S (1962): An abbreviation of the WAIS for clinical use. J Clin Psychology 18:77-79

Smith A (1973): Symbol Digit Modalities Test: Manual. Los Angeles, Western Psychological Services

Spitzer RL, Williams JBW, Gibbon M, First MB (1992): The structured clinical interview for DSM-III-R (SCID). I History, rationale, and description. Arch Gen Psychiatry 49:630-636

Thiemann S, Csernansky JG, Berger PA (1987): Rating Scales in research: The case of negative symptoms. Psychiatry Res 20:47-55

Wechsler DA (1945): A standardized memory scale for clinical use. J Psychology 19:87-95 